Research Paper

\title{
Role of Sirolimus in renal tubular apoptosis in response to unilateral ureteral obstruction
}

\author{
Mei Yang1, Yang-yang Zhuang1, Wei-wei Wang1, Hai-ping Zhu1, Yan-jie Zhang1, Sao-ling Zheng2, \\ Yi-Rrong Yang ${ }^{2}$, Bi-Cheng Chen ${ }^{3}$, Peng $\mathrm{Xia}^{2}{ }^{\varpi}$, Yan Zhang ${ }^{2}$ \\ 1. Department of Intensive Care Unit, the First Affiliated Hospital of Wenzhou Medical University, Wenzhou, Zhejiang Province, China 325015 \\ 2. Transplantation centre, the First Affiliated Hospital of Wenzhou Medical University, Wenzhou, Zhejiang Province, China 325015 \\ 3. Zhejiang Provincial Top Key Discipline in Surgery, Wenzhou Key Laboratory of Surgery, Department of Surgery, The First Affiliated Hospital of Wenzhou \\ Medical University, Wenzhou, Zhejiang Province 325015, China \\ $\triangle$ Corresponding authors: Peng Xia, MD, Transplantation centre, the First Affiliated Hospital of Wenzhou Medical University, Wenzhou, Zhejiang, China \\ 325015. Phone: +86-13857753169, E-mail: pengxia602@163.com and Yan Zhang, MD, PHD, Transplantation centre, the First Affiliated Hospital of Wenzhou \\ Medical University, Wenzhou, Zhejiang, China 325015. Phone: +86-15858583023, E-mail: biobabry@163.com \\ (c) Ivyspring International Publisher. This is an open access article distributed under the terms of the Creative Commons Attribution (CC BY-NC) license \\ (https://creativecommons.org/licenses/by-nc/4.0/). See http://ivyspring.com/terms for full terms and conditions.
}

Received: 2018.04.28; Accepted: 2018.07.26; Published: 2018.09.07

\begin{abstract}
Renal tubule cell apoptosis plays a pivotal role in the progression of chronic renal diseases. The previous study indicates that Sirolimus is effective on unilateral ureteral obstruction (UUO)-induced renal fibrosis. However, the role of Sirolimus in renal tubular apoptosis induced by UUO has not yet been addressed. The aim of this study was to determine the role of Sirolimus in renal tubular apoptosis induced by UUO. Male Sprague-Dawley rats were divided into three groups, sham-operated rats, and after which unilateral ureteral obstruction (UUO) was performed: non-treated and sirolimus-treated (1 mg/kg). After 4, 7 and $14 \mathrm{~d}$, animals were sacrificed and blood, kidney tissue samples were collected for analyses. Histologic changes and interstitial collagen were determined microscopically following HE and Masson's trichrome staining. The expression of PCNA was investigated using immunohistochemistry and the expression of $\mathrm{Bcl}-2$, Bax, caspase-9, and caspase-3 were investigated using Western blot in each group. Tubular apoptotic cell deaths were assessed by terminal deoxynucleotidyl transferase-mediated dUTP nick-end labeling (TUNEL) assay. Sirolimus administration resulted in a significant reduction in tubulointerstitial fibrosis scores. After UUO, there was an increase in tubular and interstitial apoptosis in untreated controls as compared to Sirolimus treatment rats $(P<0.05)$. In addition, the expression of PCNA, Bcl-2, Bax, caspase-9, and caspase- 3 in obstructed kidney was characterized by immunohistochemistry and Western blot analyses demonstrating that sirolimus treatment significantly reduced PCNA, Bax, caspase- 9 and cleaved caspase- 3 expression compared to those observed in controls $(P<0.05)$, whereas, $\mathrm{Bcl}-2$ in the obstructed kidney were decreased in untreated controls compared to Sirolimus treatment rats subjected to the same time course of obstruction $(P<0.05)$. We demonstrated a marked renoprotective effect of sirolimus by inhibition of UUO-induced renal tubular apoptosis in vivo.
\end{abstract}

Key words: Urinary tract obstruction; apoptosis; proliferation; renoprotection; sirolimus

\section{Introduction}

Progressive renal fibrosis is the final common manifestation of various chronic kidney diseases (CKD) resulting in renal atrophy and end-stage renal failure. Development of fibrotic kidney disease is refractory and effective therapy is not yet available. The characteristics of renal fibrosis comprise decreases in renal function, increased interstitial fibrosis, tubular apoptosis, and cellular infiltration [1-3]. Unilateral ureteral obstruction (UUO) is a representative model of tubulointerstitial renal fibrosis that has many readily quantifiable cellular and molecular events, such as inflammation and apoptosis [4]. Ample evidence suggests that destruction of renal tubular cells by apoptosis resulting from urinary tract obstruction leads to tubular atrophy, one of the hall-marks of obstructive nephropathy $[5,6]$. The regulation of apoptosis in the obstructed kidney is of considerable interest [7]. 
In recent years, great efforts have been made to gain further insight into the mechanisms of apoptosis in obstructed kidney and several molecules with pro-apoptosis properties, such as bcl-2 and p53, have been proposed $[7,8]$. Thus, suppression of apoptosis signaling has been included in several therapeutic approaches for preventing renal fibrosis $[9,10]$.

Sirolimus (also known as Rapamycin) is an antifungal agent originally purified from Streptomyces hygroscopicus [11]. It was later found to have potent immunosuppressive effects and has been used for many years as a component of antirejection therapy for recipients of organ transplants [12, 13]. The antifibrotic effects of mTOR inhibition have recently been reported in several rat models of chronic kidney disease, including diabetic nephropathy, chronic glomerulosclerosis, and tubulointerstitial fibrosis [14-18]. Likewise, rapamycin prevented extracellular matrix deposition in CCL4-induced liver fibrosis [19], attenuated liver cirrhosis progression [20], prevented bleomycin-induced pulmonary fibrosis [21]. Thus, sirolimus could have a pivotal role in disease states characterized by fibrogenesis and may emerge as a promising pharmacotherapeutic target for anti-fibrotic treatment.

However, the potential involvement of sirolimus in renal tubular apoptosis has so far not been investigated. In the present study, we hypothesized that sirolimus also attenuates renal tubular apoptosis and the development of tubulointerstitial fibrosis in the kidney. Here, we examined whether sirolimus plays an important role in renal tubular apoptosis in the UUO model.

\section{Materials and Methods}

Male Sprague-Dawley rats weighing between 250-300g were purchased from Beijing Vital River Laboratory. Rats had access to standard rat chow and water ad libitum and were maintained following conditions established by the Guide for the Care and Use of Laboratory Animals. During the entire experiment rats were kept in individual metabolic cages, with a 12-h artificial light-dark cycle, a temperature of $21 \pm 2^{\circ} \mathrm{C}$, and a humidity of $55 \pm 2 \%$. Rats were allowed to acclimatize to the cages for 3 days before surgery.

Before surgery, the rats were anesthetized with an intra-peritoneal (ip) injection of $60 \mathrm{mg} / \mathrm{kg}$ sodium pentobarbital (Merial, Hallbergmoos, Germany), and during surgery, they were placed on a heated table to maintain rectal temperature at $37-38^{\circ} \mathrm{C}$. UUO were established as previously described [22]. In brief, the left ureter was exposed and a 5-0 silk ligature occluded the midportion of the ureter. After surgery, the rats regained consciousness and were placed in metabolic cages. Rats were allocated to the protocols indicated below. Age- and time-matched sham-operated controls were prepared and observed in parallel with UUO group in the following protocols.

Protocol $1 \quad(\mathrm{n}=18)$ : Sprague-Dawley rats underwent UUO.

Protocol 2 (n=18): Sprague-Dawley rats underwent UUO treated with sirolimus $(2 \mathrm{mg} / \mathrm{kg}$ body weight, Wyeth Pharmaceuticals Company, Guayama, Puerto Rico, USA).

Protocol $3(\mathrm{n}=18)$ : sham-operated rats (Sham).

Rats ( $n=6$ per group) were sacrificed 4 , 7and 14 days after surgery. After anesthesia with sodium pentobarbital $(60 \mathrm{mg} / \mathrm{kg})$, a laparotomy was performed and the abdominal aorta was cannulated with a 23-gauge needle, and then the organs were perfused with ice-cold lactated Ringer solution. Kidney were removed, cut in thirds, and then fixed for $20 \mathrm{~h}$ in $3.75 \%$ paraformaldehyde in Soerensen's phosphate buffer and embedded in paraffin for histological examination, snap frozen in isopentane $\left(-40^{\circ} \mathrm{C}\right)$ for cryostat sectioning, or frozen in liquid nitrogen and stored at $-80^{\circ} \mathrm{C}$ for protein chemistry analysis.

\section{Histological analysis}

Renal tissue sections were stained with hematoxylin and eosin and Masson's trichrome for histological assessment. Kidneys were routinely fixed in $4 \%$ phosphate-buffered paraformaldehyde and paraffin embedded. Tissue sections at $5 \mu \mathrm{m}$ were obtained. Paraffin wax was removed with xylene, and sections were rehydrated with ethanol. After washing, the sections were stained with hematoxylin and eosin. Renal injury index including inflammatory, cell infiltration, interstitial fibrosis, interstitial edema, cell vacuolar degeneration, tubular atrophy, and tubular expansion were measured to assess the renal interstitial lesions. Ten different fields were selected to estimate the level of renal injury index with HE staining using bio-image analysis system (Bio-Profile). Each parameter was evaluated and given a score from 0 to $4+,(0$, no changes; $1+$, changes affecting $5-25 \%$ of the sample; $2+$, changes affecting $25-50 \%$; $3+$, changes affecting $50-75 \%$; $4+$, changes affecting $75-100 \%$ ). For analyzing the degree of tubulointerstitial collagen deposition, sections were stained with Masson trichrome. Twenty cortical tubulointerstitial fields that were randomly selected at $\times 400$ magnification were assessed in each rat, and the density of trichrome-positive signals was analyzed by bio-image analysis system (Bio-Profile). All the samples were semi-quantitatively or quantitatively assessed by two independent investigators in a blinded manner. 


\section{Immunohistochemistry (IHC)}

The expression of PCNA (diluted 1:300; Abcam, USA) were assessed in paraffin-embedded tissue sections. Immunohistochemical staining was performed as described previously [23]. Briefly, paraffin-embedded sections were dewaxed (or frozen sections were hydrated) and microwave oven heated in $0.1 \mathrm{M}$ sodium citrate buffer for $12 \mathrm{~min}$. After the serum block, sections were incubated with primary antibodies in PBS with $3 \%$ BSA overnight at $4^{\circ} \mathrm{C}$. Sections were washed, and the primary antibodies were detected using the $A B C$ method and developed with 3,3-diaminobenzidine (DAB) to produce a specific antigen brown color.

\section{Western blot Analysis}

Kidney tissues were lysed in RIPA buffer, run on a 10\% SDS-polyacrylamide electrophoresis gel and transferred onto a nitrocellulose membrane (Hybond C Extra, Amershan Biosciences, Little Chalfon, USA). The membrane was incubated in a blocking buffer A (PBS, 5\% nonfat milk and $0.1 \%$ Tween-20) and incubated overnight at $4{ }^{\circ} \mathrm{C}$ with primary rabbit anti-rat Bax (diluted 1:300; Abcam, USA), Bcl-2 (diluted 1:300; Abcam, USA), Caspase-3 (diluted 1:200; Abcam, USA), Caspase-8 (diluted 1:300; Abcam, USA) and Caspase-9 (diluted 1:200; Abcam, USA) antibody. Then the membrane was washed once for 15 min and twice for five min in PBS, followed by a peroxidase-conjugated sheep anti-rabbit IgG (Santa Cruz Biotechnology) at a 1:10000 dilution. At last, the membrane was developed with a chemiluminescent agent (ECL). Each membrane was stripped and probed with mouse primary anti- $\beta$-actin antibody (Sigma, USA) to confirm and estimate the loading and the transfer. We used a bio-image analysis system (Bio-Rad, USA) to analyze the bands.

\section{TUNEL assay}

TUNEL assays were performed to detect DNA strand breaks using a commercial kit following the instructions provided by the manufacturer's (Roche's In Situ Cell Death Detection Kit, Fluorescein (Indianapolis, IN)) recommendations. Briefly, $15-\mu \mathrm{m}$-thick sections of renal ( $\mathrm{n}=6$ per group) were mounted onto Silane-coated glass slides. Slides were deparaffinized, rehydrated, put into $10 \mathrm{mM}$ citrate $\mathrm{pH}$ 6 in a $95^{\circ}$ water bath for 30 minutes for permeabilization and further digested with $1 \mu \mathrm{g} / \mathrm{ml}$ proteinase $\mathrm{K}$ for 10 minutes at $37^{\circ}$. TUNEL reagents were applied to the slides according to the manufacturer's instructions. Then they were mounted with DAPI Vectashield (Vector Laboratories). Controls for this procedure included a slide where the TdT enzyme was omitted and another where the slide was pretreated with DNAse I before the normal TUNEL procedure. Photographs of sections were captured using CCD camera (Leica DC300F). The number of apoptotic nuclei was counted in four different fields and mean was found by using the image analysis software 'Leica Qwin'. Percentage of TUNEL positive cells was calculated on the number of TUNEL positive cells out of 100 total cells that were counted. Student's t-test was used to determine statistical significance levels $(P \leq 0.05)$.

\section{Statistical Analysis}

Results were assessed using a one-way ANOVA for comparisons between groups. Differences were assessed using the Bonferroni pos-test, with $P<0.05$ considered indicative of significant differences. Data are expressed as the mean \pm standard error of the mean (SEM).

\section{Results}

\section{Sirolimus treatment protects against renal fibrosis, tubular dilation and atrophy induced by UUO in a murine UUO model.}

To assess the effects of sirolimus on renal fibrosis and tubular atrophy, the kidneys of male Sprague-Dawley rats subjected to UUO or sham operation and treat daily with sirolimus $(2 \mathrm{mg} / \mathrm{kg} / \mathrm{d})$ were examined for histopathology (Fig. 1). HE stainings shows that UUO renal histology displays a spectrum of changes including tubular dilation and interstitial edema in the early stage, and tubular atrophy and interstitial fibrosis in the later phase. Histological analysis showed a higher percentage of fibrosis in the animals subjected only to UUO when compared to sirolimus-treated animals (Fig. 5). Indeed, sirolimus-treated animals showed an impressive two-fold decrease in the percentage of fibrosis (Fig. 5) $(P<0.05)$. Masson's trichrome stain of representative kidney sections also demonstrates increased collagen deposition within the tubulointerstitium 4, 7 and 14 days in rats after undergoing UUO (Fig. 2, D, E and F, respectively). However, treat daily with sirolimus suppressed the tubulointerstitial collagen deposition at the same time course post-operation (Fig. 2, G, H and I, respectively) $(P<0.05)$. No gross alterations were observed in those sham-operated rats (Fig. 2, A, B and C, respectively).

Tubular atrophy progressed in a time-dependent manner after undergoing UUO. In parallel with the interstitial expansion, tubular atrophy became the dominant pathologic change of end-stage of UUO kidneys. Treatment with sirolimus obviously retarded this progression as shown in Figure 1. 

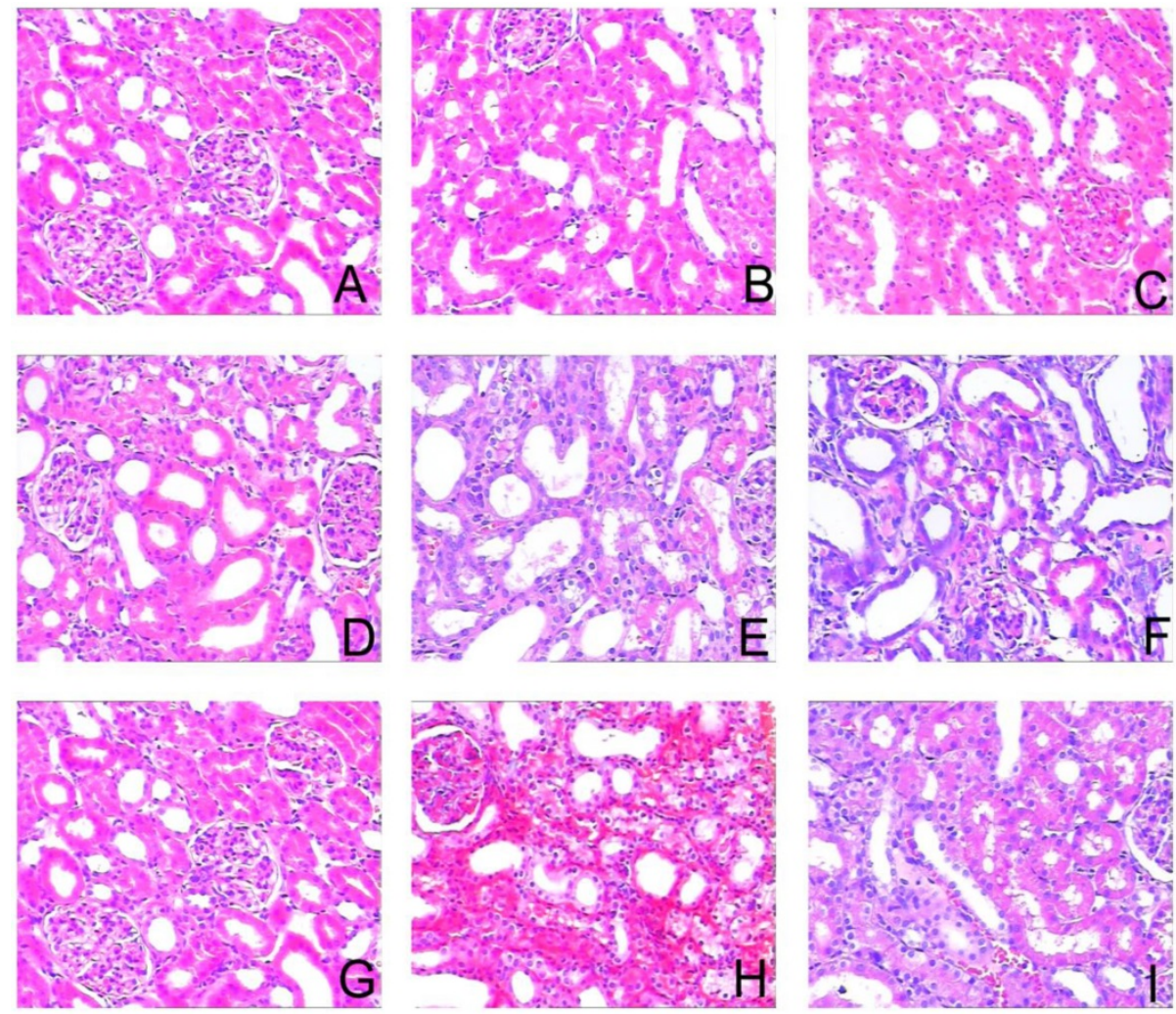

Figure 1. Sirolimus attenuated the histological changes in the obstructed kidney induced by UUO. Representative hematoxylin-eosin staining micrographs of (A, B, C) Sham group, (D, E, F) UUO group and (G, H, I) Sirolimus group. Original magnification $\times 200$.
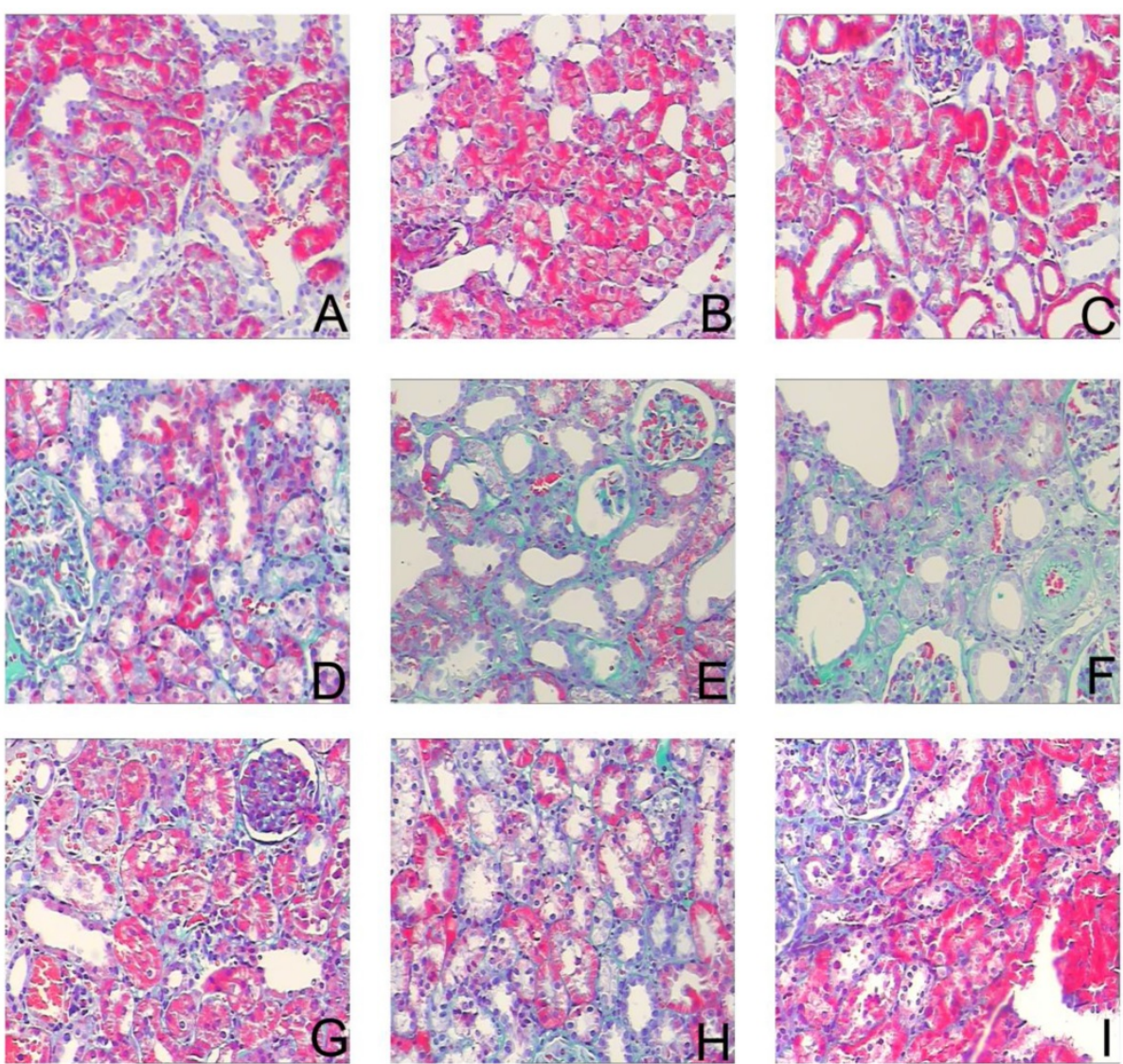

Figure 2. Sirolimus attenuated the interstitial collagen deposition in the obstructed kidney induced by UUO. Representative Masson's trichrome staining micrographs of (A, B, C) Sham group, (D, E, F) UUO group and (G, H, I) Sirolimus group. Original magnification $\times 200$. 


\section{Sirolimus treatment decrease tubular proliferation and apoptosis induced by UUO.}

To investigate whether the Sirolimus treatment could moderate tubular cell proliferation and apoptosis in post-obstructed kidneys, we examined the changes of proliferating tubular cells, identified as PCNA-positive nuclei and apoptotic bodies, marked as an in situ end-labeled DNA fragment with the TUNEL method. Compared with sham rats, proliferating tubular cells were increased significantly throughout the whole experimental period (Fig. 3). In Sirolimus treatment group, the prolifer-ative activity was significantly decreased in each time course (Fig. 5) $(P<0.05)$. To determine whether Sirolimus can protect renal tubular epithelial cells from apoptosis, the number of tubular apoptotic bodies were counted and the results are summarized in Figure 4. In parallel with the fibrosis-related index described earlier, tubular apoptosis was activated after UUO operation and progressively increased during the entire two-week course (Figure 4). Sirolimus treatment suppressed the tubular apoptosis at the same time point after UUO (Fig. 5) $(P<0.05)$.
Sirolimus moderate expression of Bcl-2, Bax, caspase-3, caspase- 8 and caspase- 9 induced by UUO.

We examined kidney tissue lysates obtained from sham-operated control rat and from rat after undergoing UUO and treat with Sirolimus. Levels of Bcl-2, Bax, caspase-3, caspase- 8 and caspase- 9 were assessed by Western blot analysis and representative blots are shown in Fig. 6, A, B, C, D and E. Quantitative analysis by densitometry shows significant increases in the expression of Bax, caspase-3, caspase- 8 and caspase-9 in the kidneys of rat at day 4 following UUO (Fig. 6), and further increased at day 7 and 14 after undergoing UUO (Fig. 6) compared with control sham-operated rat. In contrast, in rats treat with Sirolimus significant reductions in the Bax, caspase- 3 , caspase- 8 and caspase- 9 expression were observed at both 4,7 and 14 days after UUO compared with UUO rats at the same time point $(P<0.05)$. The expression of Bcl-2 in the obstructed kidney was significantly decreased in a time-dependent manner (Fig. 6). The administration of Sirolimus significantly increase of Bcl-2 expression compared to UUO rats at the same time point (Fig. 6) $(P<0.05)$.
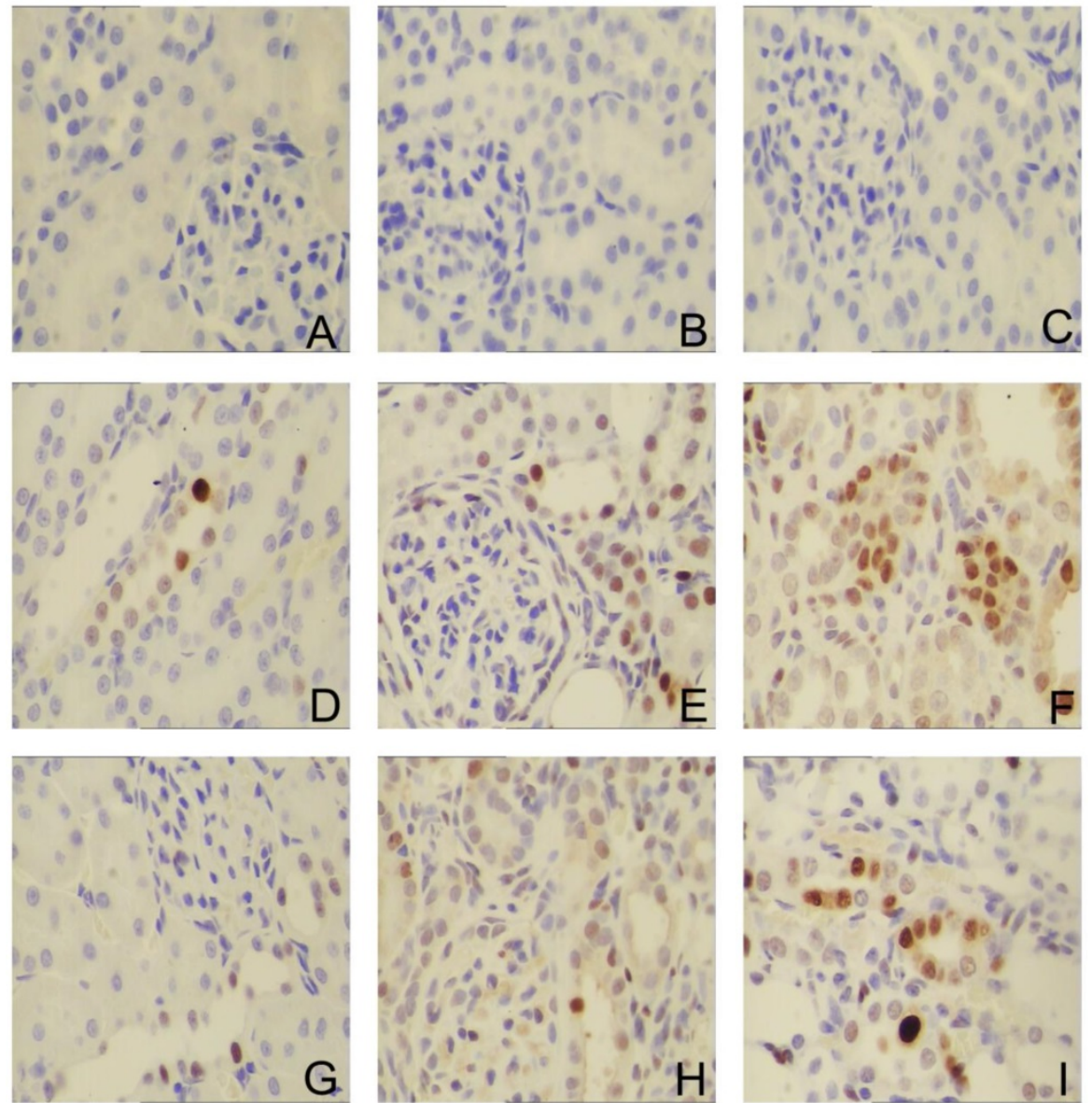

Figure 3. Sirolimus suppressed the expression of PCNA in the obstructed kidney induced by UUO. Representative micrographs of (A, B, C) Sham group, (D, E, F) UUO group and $(G, H, I)$ Sirolimus group. Original magnification $\times 400$. 

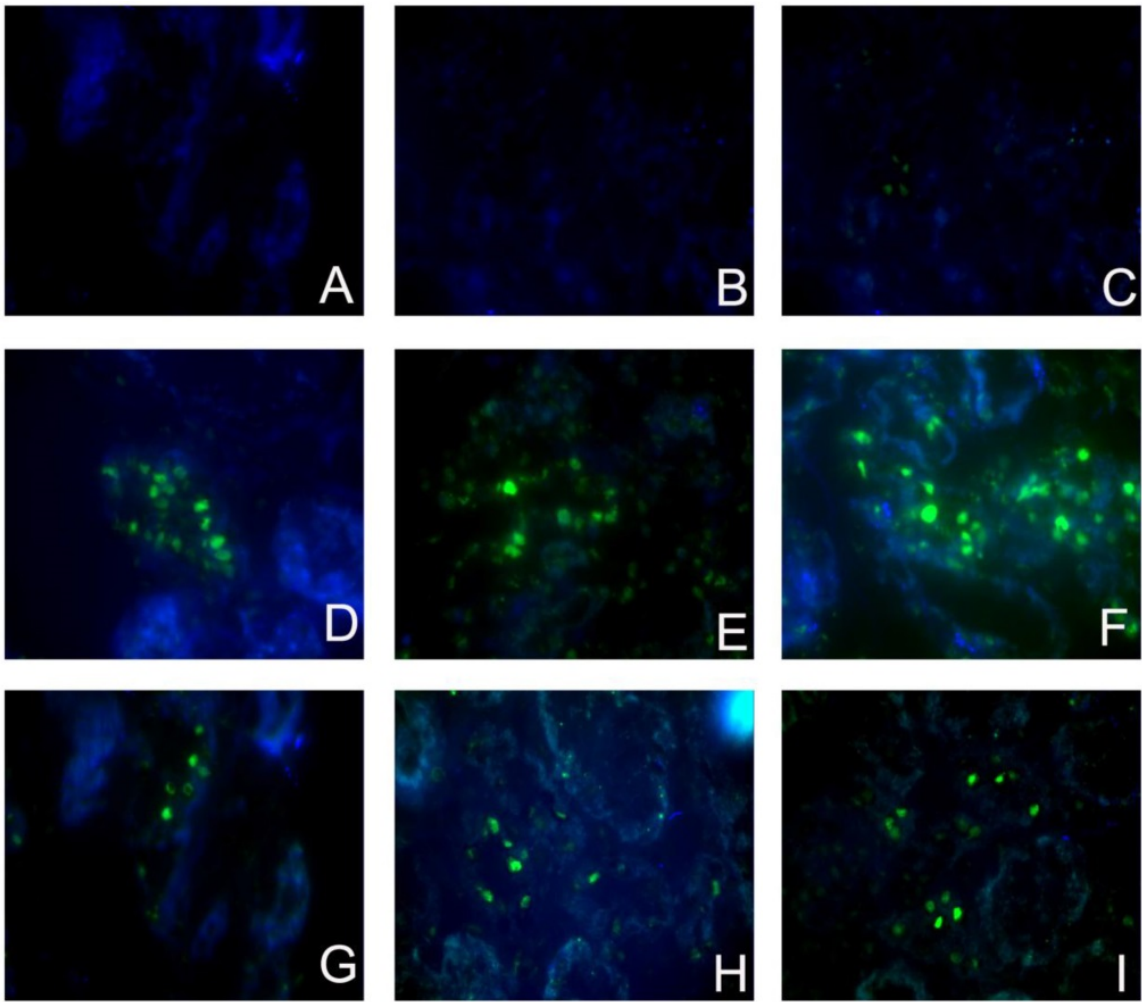

Figure 4. Sirolimus decreased the TUNEL+ cells in the obstructed kidney induced by UUO. Representative micrographs of (A, B, C) Sham group, (D, E, F) UUO group and (G, $\mathrm{H}, \mathrm{I})$ Sirolimus group. Original magnification $\times 400$.

\section{A}

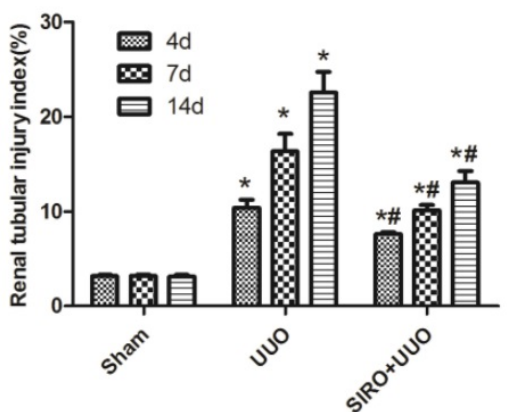

C

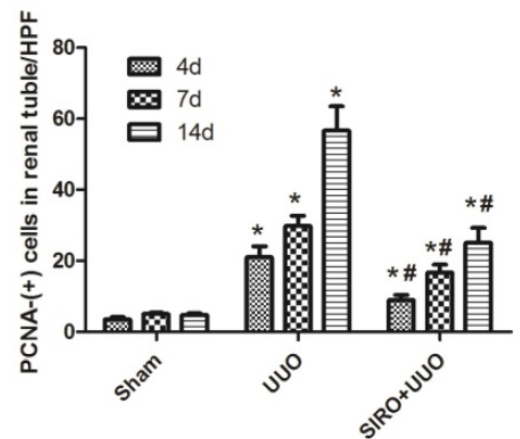

B

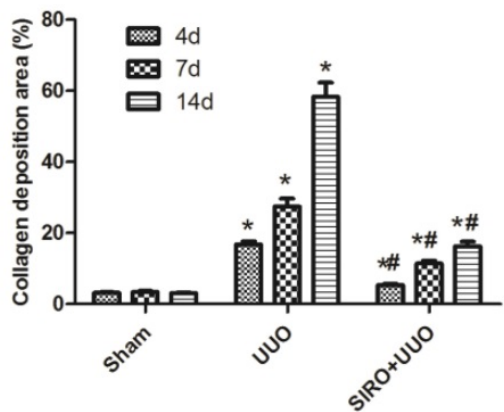

D

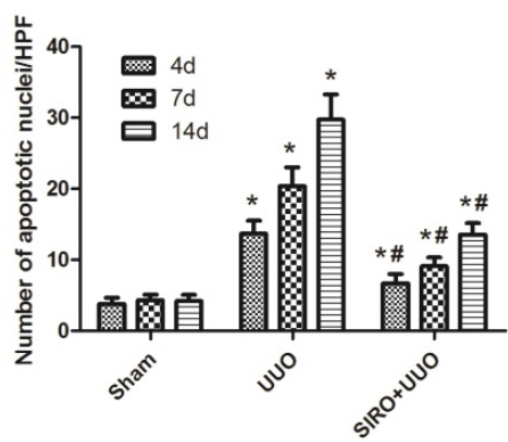

Figure 5. Comparison of the expression for Renal tubular injury index, Collagen deposition area, PCNA-(+) cells in renal tubular, and number of apoptotic nuclei in each group. A. Renal tubular injury index (\%) in each group. B. Collagen deposition area (\%) in each group. C. PCNA-(+) cells in renal tubular/HPF in each group. D. Number of apoptotic nuclei/HPF in each group. $* P<0.05$ in comparison with Sham group. $\# P<0.05$ in comparison with UUO group 

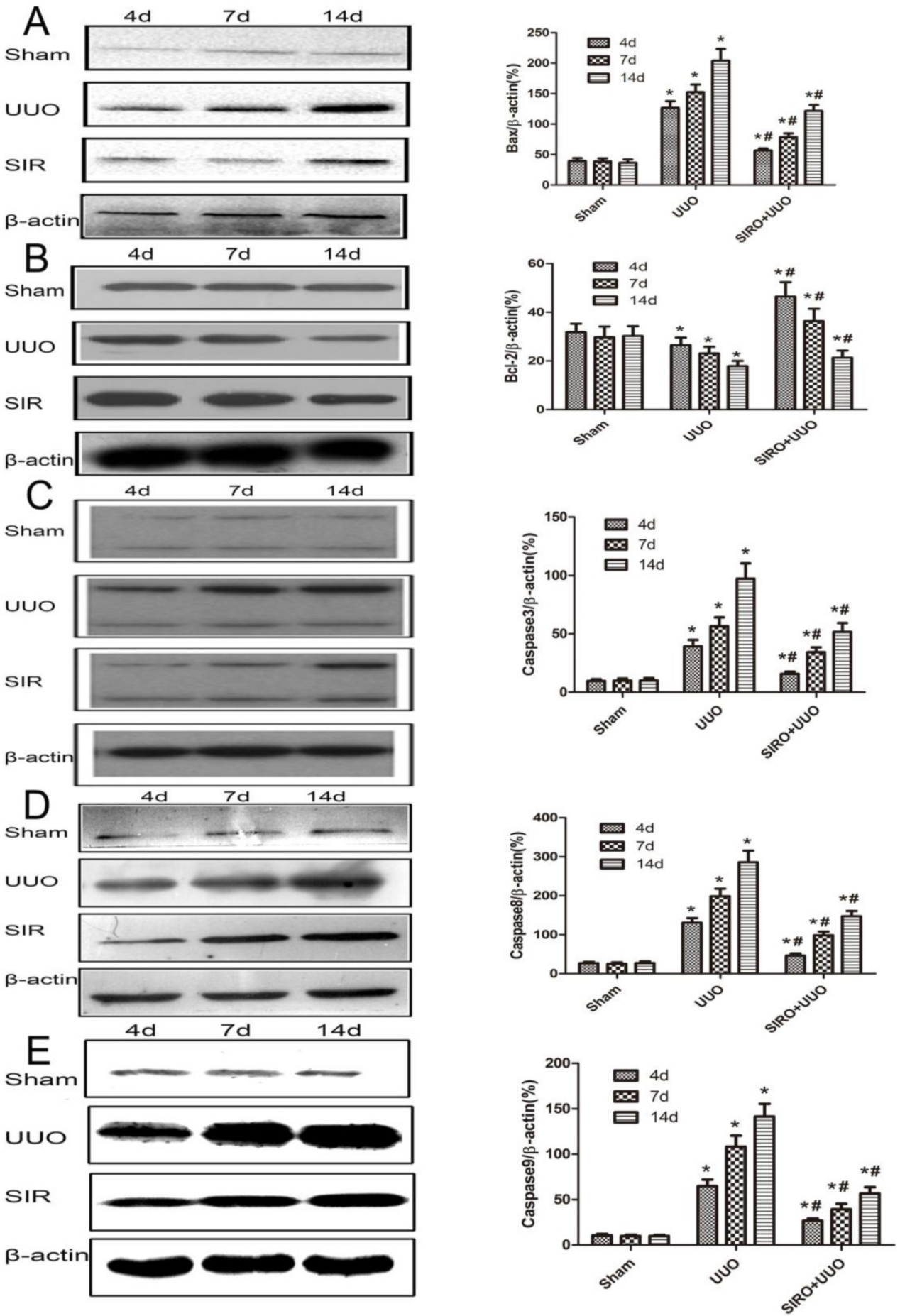

Figure 6. Comparison of the expression for Bcl-2, Bax, caspase-3, caspase-8, and caspase- 9 in each group. The same blot was stripped and reprobed with actin to confirm equal loading. A. Sirolimus suppressed the expresion of Bax in the obstructed kidney assessed by Western blot assay. B. Sirolimus increased the expresion of Bcl-2 in the obstructed kidney assessed by Western blot assay. C. Sirolimus suppressed the expresion of Caspase 3 in the obstructed kidney assessed by Western blot assay. D. Sirolimus suppressed the expresion of Caspase8 in the obstructed kidney assessed by Western blot assay. E. Sirolimus suppressed the expresion of Caspase 9 in the obstructed kidney assessed by Western blot assay. $* P<0.05$ in comparison with Sham group. $\# P<0.05$ in comparison with UUO group.

\section{Discussion}

Chronic kidney disease (CKD) is the result of various lesions to the kidney, affecting approximately $10 \%$ of the normal population. Unchecked progression of CKD without fail leads to ESRD and the requirement for renal replacement therapy (renal transplantation or dialysis). Given the high prevalence of CKD and cost of replacement therapies for ESRD, any treatment that halts or slows the progression of renal fibrosis has the possibilities to provide a gigantic medical, social and economical benefit. Renal fibrosis, characterized by glomerulosclerosis and tubulointerstitial fibrosis, is the final manifestation of CKD [24]. 
Although various diseases such as glomerulonephritis; metabolic diseases, including diabetes mellitus and atherosclerosis; obstructive nephropathy; interstitial nephritis; and cystic nephropathies, including polycystic kidney disease, can be the major causes of CKD, renal fibrosis is always the common terminal result of CKD [25, 26]. The mechanisms underlying the progression of renal disease to end-stage renal failure are not well understood.

With end-stage renal disease, renal cells are replaced by fibrous tissue given to the sclerotic changes observed in the glomeruli and the interstitium. The transition from renal growth and hypercellularity to cell deletion and atrophy raises the question as to which process is responsible for cell loss [27].

Apoptosis, or programmed cell death, is a likely mechanism involved in the progression loss of renal cells during the course of renal fibrosis. Apoptosis is a particular type of cell death, which has several distinguishing features from necrosis, and is often referred to as physiological or programmed cell death. It is also play an important role in the regulation of renal cell number in both healthy and diseased kidneys [28, 29].

Unilateral ureteral obstruction (UUO), a well characterized experimental model of renal tubulointerstitial fibrosis, results in renal functional loss and morphological changes including hydronephrosis, infiltration of leukocytes, tubular atrophy, and dilation, as well as increased interstitial fibrosis [1]. An accumulating body of evidence suggests that tubular cell apoptosis result to fibrotic kidney changes that occur in conjunction with ureteral obstruction [29, 30]. Stretch, ischemia, and oxidative stress followed by ureteral obstruction are primary causes of tubular cell apoptosis [4]. Increased apoptosis activate cellular infiltration, interstitial cell proliferation, and interstitial fibrosis $[29,30]$.

The extent of tubular apoptosis in animal models of ureteric obstruction correlates with the severity of tubular injury and tubulointerstitial fibrosis [30-32]. Also, inhibition of initial tubular cell apoptosis by either neutralizing the activity of apoptosis-inducing molecules or supplementing with prosurvival factors effectively prevents inflammation and attenuates progression to fibrosis in the UUO model [33, 34]. These researches provide evidence for an apparent interplay between early apoptosis and subsequent fibrosis, and the apoptosis could be an early event that occurs before the onset of frank fibrosis.

Apoptosis can be triggered either by the extrinsic pathway, which involves the activation of death receptors on the cell surface, or the intrinsic mitochondrial pathway, which involves the release of several proapoptotic factors from the mitochondria to the cytosol, thereby inducing caspase activations [35, 36]. There is evidence that this intrinsic mitochondrial pathway is involved in stretch-induced tubular cell apoptosis. Tubular BAX protein expression increased over time after UUO, while there was a decrease in Bcl-2 expression [37].

Bcl-2 was the first gene shown to be specifically involved in the process of physiological cell death. It can inhibit apoptosis of many cells triggered by diverse aetiology. Bax, having a structural similarity to $\mathrm{Bcl}-2$, is able to antagonise the protection offered by Bcl-2 [38].

Under various circumstances, the activity of the Bcl-2 protein may be regulated through caspase cleavage [39]. Caspases have been considered to be attractive potential targets for treatment of diseases because of their crucial role in apoptosis and the appealing prospect of small molecule inhibitor therapy [40]. Caspases play a key role in the modulation of apoptosis and apoptotic pathways, on the one hand, caspase-8, an initiator caspase [41] which mediates Fas induced death pathway, and caspase-9, which is vital for the mitochondrial mediated death [42]. On the other hand, caspase- 8 cleaves BID to tBID which translocate to mitochondria and release cytochrome $c$ [43]. Caspase-3, the effector caspase, is important for both extrinsic and intrinsic pathway with well documented role in the regulation of neutrophil apoptosis [44].

In this study, Bcl-2 and Bax proteins showed an inverse correlation. Bax protein increased with time as Bcl-2 levels fell during UUO. These were consistent with and may account for the changes of transient proliferation and progressive apoptosis after UUO. As the previous studies shows that apoptosis was more common in tubules highly expressing Bax protein. The results of this study suggest that as regulators of apoptosis Bcl-2 and Bax may play key roles in the process of tubular atrophy and interstitial fibrosis during UUO.

Cell proliferation and apoptosis are obligatory physiological companions in the kidney. Proliferating cell nuclear antigen (PCNA), a 36-kDa DNA polymerase protein, has an essential role in cellular synthesis and cell cycle progression [45]. It may express a compensatory proliferative response to the loss of cells through programmed cell death in an attempt to maintain renal structural integrity. Proliferation of the obstructed kidney has previously been studied [46]. An increase in proliferation of both tubular and interstitial cells, with different time courses, was demonstrated. Our data reveal that the number of PCNA-positive nuclei in the UUO group 
was significantly higher than that in the control group, The administration of sirolimus significantly decreased the number of PCNA-positive nuclei compared to UUO rats at the same time point $(\mathrm{P}<0.05)$.

Sirolimus (also known as Rapamycin) was isolated from a soil bacterium in 1975 [13]. The discovery of rapamycin led to the identification and cloning of mammalian target of rapamycin (mTOR), a serine/threonine kinase, in 1994 [47]. Rapamycin is a potent, specific inhibitor of mTOR and does not inhibit any kinase other than mTOR [13]. Because of its high specificity for mTOR, rapamycin has been very useful in establishing the role of $\mathrm{MTOR}$ in cell biology and in the pathogenesis of disease [47, 48]. Although initially isolated as an antifungal agent, rapamycin was later found to have potent immunosuppressive effects and has been used for many years as a component of anti rejection therapy for recipients of organ transplants [49].

Structural analysis of rapamycin reveals that it is an analogue of the macrolide antibiotic FK506. Similar to FK506, rapamycin also has immunosuppressive effects [11]. Rapamycin analogs with improved pharmaceutical properties have been used clinically to inhibit both host rejection following organ transplantation and the restenosis of coronary arteries after angioplasty [50].

Additionally, sirolimus proved to have potent anti-proliferative actions in the experimental models of bleomycin-induced pulmonary fibrosis [21], CCL4-induced liver fibrosis [19], cirrhosis progression [20], and carbon tetrachloride-induced hepatic fibrosis in rats [51]. In the meantime sirolimus exhibit anti-fibrotic properties in several rat models of chronic kidney disease, including diabetic nephropathy, chronic glomerulosclerosis, and tubulointerstitial fibrosis [14-18]. In UUO models, sirolimus was demonstrated to inhibit interstitial macrophages and myofibroblasts [17], reduce renal hypoxia, interstitial inflammation [18], delay the progression of tubulointerstitial renal fibrosis [15], but whether sirolimus affects renal tubular apoptosis after urinary tract obstruction is rarely reported.

The results of this study suggest that Sirolimus protects against renal fibrosis, tubular dilation and atrophy induced by UUO. Sirolimus treatment significantly decrease in the percentage of fibrosis, suppressed the tubulointerstitial collagen deposition compared with UUO group at the same time point. Sirolimus treatment effectively suppressed expression of PCNA and tubular apoptosis induced by UUO. Furthermore, Sirolimus treatment significantly decrease the expression of Bax, caspase-3, caspase-8 and caspase- 9 in response to ureteral obstruction.
However, Sirolimus treatment significantly increase the expression of Bcl-2 compared with UUO group at the same time point.

In conclusion, our study demonstrates that the balance between proliferation and apoptosis is of critical importance in the process of tubulointerstitial fibrosis in response to ureteral obstruction, which is partly regulated by Bcl-2 and Bax proteins. Sirolimus treatment moderate tubular proliferation and apoptosis induced by UUO. In addition, Sirolimus moderate expression of Bcl-2, Bax, caspase-3, caspase- 8 and caspase- 9 in response to ureteral obstruction. Taken together, our study confirms that sirolimus protects the obstructed kidney by inhibiting renal tubular apoptosis.

\section{Acknowledgements}

Supported by Natural Science Foundation of China: 81505382; Zhejiang Provincial Natural Science Foundation: LQ16H100002; LY16H050007; Zhejiang Province Science and Technology Hall Foundation: 2015C33186; Wenzhou Science and Technology Foundation: Y20170181; Y20170256

\section{Competing Interests}

The authors have declared that no competing interest exists.

\section{References}

1. Klahr S, Morrissey J. Obstructive nephropathy and renal fibrosis. American journal of physiology Renal physiology. 2002; 283: F861-75.

2. Neilson EG. Mechanisms of disease: Fibroblasts--a new look at an old problem. Nature clinical practice Nephrology. 2006; 2: 101-8.

3. Zeisberg M, Neilson EG. Mechanisms of tubulointerstitial fibrosis. J Am Soc Nephrol. 2010; 21: 1819-34.

4. Mei W, Peng Z, Lu M, Liu C, Deng Z, Xiao Y, et al. Peroxiredoxin 1 inhibits the oxidative stress induced apoptosis in renal tubulointerstitial fibrosis. Nephrology. 2015; 20: 832-42.

5. Chevalier RL. Pathogenesis of renal injury in obstructive uropathy. Current opinion in pediatrics. 2006; 18: 153-60.

6. Truong LD, Gaber L, Eknoyan G. Obstructive uropathy. Contributions to nephrology. 2011; 169: 311-26.

7. Chevalier RL, Smith CD, Wolstenholme J, Krajewski S, Reed JC. Chronic ureteral obstruction in the rat suppresses renal tubular Bcl-2 and stimulates apoptosis. Experimental nephrology. 2000; 8: 115-22.

8. Leri A, Claudio PP, Li Q, Wang X, Reiss K, Wang S, et al. Stretch-mediated release of angiotensin II induces myocyte apoptosis by activating p53 that enhances the local renin-angiotensin system and decreases the Bcl-2-to-Bax protein ratio in the cell. The Journal of clinical investigation. 1998; 101: 1326-42.

9. Topcu SO, Celik S, Erturhan S, Erbagci A, Yagci F, Ucak R. Verapamil prevents the apoptotic and hemodynamic changes in response to unilateral ureteral obstruction. International journal of urology : official journal of the Japanese Urological Association. 2008; 15: 350-5.

10. Metcalfe PD, Leslie JA, Campbell MT, Meldrum DR, Hile KL, Meldrum KK. Testosterone exacerbates obstructive renal injury by stimulating TNF-alpha production and increasing proapoptotic and profibrotic signaling. American journal of physiology Endocrinology and metabolism. 2008; 294: E435-43.

11. Abraham RT, Wiederrecht GJ. Immunopharmacology of rapamycin. Annu Rev Immunol. 1996; 14: 483-510.

12. Sehgal SN. Rapamune (RAPA, rapamycin, sirolimus): mechanism of action immunosuppressive effect results from blockade of signal transduction and inhibition of cell cycle progression. Clinical biochemistry. 1998; 31: 335-40.

13. Sehgal SN. Sirolimus: its discovery, biological properties, and mechanism of action. Transplant Proc. 2003; 35: 7S-14S.

14. Lloberas N, Cruzado JM, Franquesa M, Herrero-Fresneda I, Torras J, Alperovich G, et al. Mammalian target of rapamycin pathway blockade slows progression of diabetic kidney disease in rats. J Am Soc Nephrol. 2006; 17: 1395-404. 
15. Wu MJ, Wen MC, Chiu YT, Chiou YY, Shu KH, Tang MJ. Rapamycin attenuates unilateral ureteral obstruction-induced renal fibrosis. Kidney international. 2006; 69: 2029-36.

16. Kramer S, Wang-Rosenke Y, Scholl V, Binder E, Loof T, Khadzhynov D, et al. Low-dose mTOR inhibition by rapamycin attenuates progression in anti-thy1-induced chronic glomerulosclerosis of the rat. American journal of physiology Renal physiology. 2008; 294: F440-9.

17. Chen G, Chen H, Wang C, Peng Y, Sun L, Liu H, et al. Rapamycin ameliorates kidney fibrosis by inhibiting the activation of mTOR signaling in interstitial macrophages and myofibroblasts. PloS one. 2012; 7: e33626.

18. Liu CF, Liu H, Fang Y, Jiang SH, Zhu JM, Ding XQ. Rapamycin reduces renal hypoxia, interstitial inflammation and fibrosis in a rat model of unilateral ureteral obstruction. Clinical and investigative medicine Medecine clinique et experimentale. 2014; 37: E142.

19. Gu L, Deng WS, Sun XF, Zhou H, Xu Q. Rapamycin ameliorates CCl4-induced liver fibrosis in mice through reciprocal regulation of the Th17/Treg cell balance. Molecular medicine reports. 2016; 14: 1153-61.

20. Neef M, Ledermann M, Saegesser H, Schneider V, Reichen J. Low-dose oral rapamycin treatment reduces fibrogenesis, improves liver function, and prolongs survival in rats with established liver cirrhosis. Journal of hepatology. 2006; 45: 786-96.

21. Simler NR, Howell DC, Marshall RP, Goldsack NR, Hasleton PS, Laurent GJ, et al. The rapamycin analogue SDZ RAD attenuates bleomycin-induced pulmonary fibrosis in rats. The European respiratory journal. 2002; 19: 1124-7.

22. Bai $Y, \mathrm{Lu} \mathrm{H}, \mathrm{Zhang} \mathrm{G}, \mathrm{Wu} \mathrm{C}$, Lin $\mathrm{C}$, Liang $\mathrm{Y}$, et al. Sedum sarmentosum Bunge extract exerts renal anti-fibrotic effects in vivo and in vitro. Life sciences. 2014; 105: 22-30.

23. Mei Y, Yangyang Z, Shuai L, Hao J, Yirong Y, Yong C, et al. Breviscapine prevents downregulation of renal water and sodium transport proteins in response to unilateral ureteral obstruction. Iranian journal of basic medical sciences. 2016; 19: 573-8.

24. Liu Y. Renal fibrosis: new insights into the pathogenesis and therapeutics. Kidney international. 2006; 69: 213-7.

25. Eitner F, Floege J. Novel insights into renal fibrosis. Current opinion in nephrology and hypertension. 2003; 12: 227-32

26. Boor P, Ostendorf T, Floege J. Renal fibrosis: novel insights into mechanisms and therapeutic targets. Nature reviews Nephrology. 2010; 6: 643-56.

27. Lee WK, Thevenod F. Novel roles for ceramides, calpains and caspases in kidney proximal tubule cell apoptosis: lessons from in vitro cadmium toxicity studies. Biochemical pharmacology. 2008; 76: 1323-32.

28. Jang HS, Padanilam BJ. Simultaneous deletion of Bax and Bak is required to prevent apoptosis and interstitial fibrosis in obstructive nephropathy. American journal of physiology Renal physiology. 2015; 309: F540-50.

29. Nilsson L, Madsen K, Krag S, Frokiaer J, Jensen BL, Norregaard R. Disruption of cyclooxygenase type 2 exacerbates apoptosis and renal damage during obstructive nephropathy. American journal of physiology Renal physiology. 2015; 309: F1035-48.

30. Docherty NG, O'Sullivan OE, Healy DA, Fitzpatrick JM, Watson RW. Evidence that inhibition of tubular cell apoptosis protects against renal damage and development of fibrosis following ureteric obstruction. American journal of physiology Renal physiology. 2006; 290: F4-13.

31. Misseri R, Meldrum KK. Mediators of fibrosis and apoptosis in obstructive uropathies. Current urology reports. 2005; 6: 140-5.

32. Razzaque MS, Ahsan N, Taguchi T. Role of apoptosis in fibrogenesis. Nephron. 2002; 90: 365-72.

33. Miyajima A, Chen J, Lawrence C, Ledbetter S, Soslow RA, Stern J, et al. Antibody to transforming growth factor-beta ameliorates tubular apoptosis in unilateral ureteral obstruction. Kidney international. 2000; 58: 2301-13.

34. Tao Y, Kim J, Faubel S, Wu JC, Falk SA, Schrier RW, et al. Caspase inhibition reduces tubular apoptosis and proliferation and slows disease progression in polycystic kidney disease. Proceedings of the National Academy of Sciences of the United States of America. 2005; 102: 6954-9.

35. Martinou JC, Youle RJ. Mitochondria in apoptosis: Bcl-2 family members and mitochondrial dynamics. Developmental cell. 2011; 21: 92-101.

36. Green DR, Kroemer G. The pathophysiology of mitochondrial cell death. Science. 2004; 305: 626-9.

37. Zhang G, Oldroyd SD, Huang LH, Yang B, Li Y, Ye R, et al. Role of apoptosis and $\mathrm{Bcl}-2 / \mathrm{Bax}$ in the development of tubulointerstitial fibrosis during experimental obstructive nephropathy. Experimental nephrology. 2001; 9: 71-80.

38. Vaux DL, Strasser A. The molecular biology of apoptosis. Proceedings of the National Academy of Sciences of the United States of America. 1996; 93: 2239-44.

39. Clem RJ, Cheng EH, Karp CL, Kirsch DG, Ueno K, Takahashi A, et al. Modulation of cell death by Bcl-XL through caspase interaction. Proceedings of the National Academy of Sciences of the United States of America. 1998; 95 : 554-9.

40. Thornberry NA, Lazebnik Y. Caspases: enemies within. Science. 1998; 281: 1312-6.

41. Tait SW, Green DR. Caspase-independent cell death: leaving the set without the final cut. Oncogene. 2008; 27: 6452-61.

42. Herold MJ, Kuss AW, Kraus C, Berberich I. Mitochondria-dependent caspase-9 activation is necessary for antigen receptor-mediated effector caspase activation and apoptosis in WEHI 231 lymphoma cells. Journal of immunology. 2002; 168: 3902-9.
43. Kantari C, Walczak H. Caspase- 8 and bid: caught in the act between death receptors and mitochondria. Biochimica et biophysica acta. 2011; 1813: 558-63.

44. McCracken JM, Allen LA. Regulation of human neutrophil apoptosis and lifespan in health and disease. Journal of cell death. 2014; 7: 15-23.

45. Strzalka W, Ziemienowicz A. Proliferating cell nuclear antigen (PCNA): a key factor in DNA replication and cell cycle regulation. Annals of botany. 2011; 107: 1127-40.

46. Miyajima A, Chen J, Poppas DP, Vaughan ED, Jr., Felsen D. Role of nitric oxide in renal tubular apoptosis of unilateral ureteral obstruction. Kidney international. 2001; 59: 1290-303.

47. Fingar DC, Blenis J. Target of rapamycin (TOR): an integrator of nutrient and growth factor signals and coordinator of cell growth and cell cycle progression. Oncogene. 2004; 23: 3151-71.

48. Hay N, Sonenberg N. Upstream and downstream of mTOR. Genes \& development. 2004; 18: 1926-45.

49. Crespo JL, Hall MN. Elucidating TOR signaling and rapamycin action: lessons from Saccharomyces cerevisiae. Microbiology and molecular biology reviews : MMBR. 2002; 66: 579-91, table of contents.

50. Garza L, Aude YW, Saucedo JF. Can we prevent in-stent restenosis? Current opinion in cardiology. 2002; 17: 518-25.

51. Zhu J, Wu J, Frizell E, Liu SL, Bashey R, Rubin R, et al. Rapamycin inhibits hepatic stellate cell proliferation in vitro and limits fibrogenesis in an in vivo model of liver fibrosis. Gastroenterology. 1999; 117: 1198-204. 